\title{
Site-Selective Immobilization of Functionalized DNA origami on Nanopatterned Teflon AF
}

\author{
Mehrnaz Shaali , Jakob G. Woller ${ }^{a}$, Patrik G. Johansson ${ }^{a}$, Jonas K. Hannestad ${ }^{a}$, \\ Laura de Battice ${ }^{a}$, Nesrine Aissaoui ${ }^{a}$, Tom Brown ${ }^{b}$, Afaf H. El-Saghee, ${ }^{b, d}$ Sergey \\ Kubatkin', Samuel Lara-Avilac, Bo Albinsson*a, and Aldo Jesorka*a \\ ${ }^{a}$ Department of Chemistry and Chemical Engineering/Physical Chemistry, Chalmers University \\ of Technology,41296, Gothenburg, Sweden \\ ${ }^{b}$ Department of Chemistry, University of Oxford, Chemistry Research Laboratory, 12 Mansfield \\ Road, Oxford, OXI 3TA, UK \\ 'Quantum Device Physics Laboratory, Chalmers University of Technology, 41296, Gothenburg, \\ Sweden \\ ${ }^{d}$ Chemistry Branch, Department of Science and Mathematics, Faculty of Petroleum and Mining \\ Engineering, Suez University, Suez 43721, Egypt.
}

To whom correspondence should be addressed: balb@chalmers.se, aldo@chalmers.se

We demonstrate the use of arrays of Teflon AF nanopillars for directing the assembly of single rectangular DNA origami scaffolds, functionalized with covalently linked fluorophore molecules, in defined positions on patterned surfaces. This was achieved by introducing Teflon AF as a non-amplified negative e-beam resist, which was exposed and chemically developed to generate arrays of hydrophobic nanopillars with a minimum feature size below $40 \mathrm{~nm}$. Binding of the DNA origami to the pillars was facilitated by porphyrin moieties as hydrophobic molecular anchors, reaching $80 \%$ coverage of the available sites. This combination of top down lithography and bottom-up self assembly is an efficient means of fabricating hierarchically structured bionanointerfaces in which the positioning of functional units is precisely controlled on the molecular level (functional units embedded in DNA origamis), and on the nanoscale at pre-designed locations on the substrate. 
DNA nanotechnology has emerged as a superb technique for the construction of large arbitrary nanoscale shapes in 2 or 3 dimensions. ${ }^{1-4}$ The origami assembly technique is based on self assembly of a long circular single stranded DNA with a multitude of individual staple strands. The final shape of the DNA origami is defined by the base sequence of the DNA strands. It is possible to modify individual bases of the staple strands, allowing for full addressability of the DNA structure with a resolution on the scale of a few nanometers. ${ }^{5-6}$ However, attachment of DNA nanostructures to technologically relevant surfaces, which is the first necessary step towards functional nanodevices, is still a challenging process. So far, most of the available methods for precise positioning of DNA are based on the application of nanopatterned silicone substrates in the presence of high concentration of $\mathrm{Mg}^{+2}$ ions. ${ }^{14-17}$ These ions are necessary to mediate the attachment by inverting the charge of the DNA so that it can bind onto the negatively charged silicone substrate. High concentrations of magnesium are, however, often detrimental for a variety of applications. It has indeed been shown that magnesium enhances the degradation of DNA, by promoting unwanted enzymatic activity.[Yang et al 2006] Magnesium can also affect the fluorescence intensity of some dye molecules,[Kring et al, 1955, J biol chem]. Alternative methods for DNA positioning onto surfaces involve the use of DNA hybridization between sticky ends of DNA origamis and complementary DNA strands fixed on a substrate [Akishiba, 2013], or the use of photochemical modification of graphene surfaces.. [Yun, Angewandte Chem, 2012,10.1002/ange.201106198] More versatile surfaces as well as convenient and facile patterning and attachment techniques for DNA origamis are therefore required in order to unlock the potential of DNA origami as addressable carrier of functional molecules in practical devices. 
Among the materials available for surface modification, Teflon Amorphous Fluoropolymer (AF) is a particularly interesting choice, as it possesses a number of attractive materials properties, including high chemical and physical stability, thermal resistance, and a low refractive index. It has one of the lowest relative dielectric constant among all solid polymers. ${ }^{18-19}$ Furthermore, it can be spin-coated on various surfaces to form ultra-thin films which are biocompatible, is optically transparent down to $\lambda=200$ $\mathrm{nm}$ and strongly hydrophobic. ${ }^{18-23}$ However, from a practical point of view, nano- and micro-patterning of Teflon AF has been a challenging process, owed to the chemical inertness of this polymer. ${ }^{24-28}$ Recently, an attractive route for micro/nanopatterning of Teflon AF by direct electron beam (e-beam) writing has been introduced, in which the exposure to e-beam radiation causes partial structural decomposition of the polymer, ebeam dose dependent reduction in film thickness (volume shrinking), as well as change in chemical composition and surface potential. ${ }^{22,29}$ However, the fabrication of isolated nanostructures to achieve higher selectivity would require a development process to discriminate exposed and unexposed areas.

By applying the perfluorinated solvent 2-butyl-tetrahydrofurane (commercial name FC75) as developing agent, we completed the lithographic procedure and thus enabled the use of Teflon AF as electron beam resist. The developer readily dissolves unexposed Teflon AF, but leaves e-beam-exposed areas unaltered on the substrate. The utility of this non-amplified negative resist is evaluated by determining the contrast and sensitivity to fabricate dense arrays of nanostructures with $30 \mathrm{~nm}$ in width and $40 \mathrm{~nm}$ in pitch as smallest feature sizes..

Here we show the utility of this non-amplified e-beam resist to fabricate dense arrays of nanostructures which are then used to direct the assembly of single DNA origami rectangles, assisted by hydrophobic porphyrin anchors attached to the DNA backbone. By 
depositing modified DNA origami, featuring ATTO-647N fluorophores covalently attached in pre-designed positions, on nanopillars of Teflon AF, we achieved positioning of the fluorophores on the surface with the precision of a few nanometers, reaching a surface coverage in the patterned areas of $\sim 80 \%$.

\section{Results}

\section{Site-selective DNA origami immobilization on Teflon AF}

In order to use hydrophobic Teflon AF surfaces as supporting substrates for hydrophilic DNA origami, the DNA strands were modified with 1 to 5 porphyrin molecules as anchoring moieties (See Supplementary information SI2). Moreover, ATTO-647N fluorophores were attached to each origami sheet in separate, defined positions.

In a first experiment, fluorophore-modified DNA origami were deposited onto plain, unexposed Teflon AF surfaces (Fig. 1a-d), and the surface coverage dependency on the number of anchoring porphyrins was investigated by means of total internal reflection fluorescence (TIRF) microscopy (Fig. 1e). This control showed that origami without porphyrin modification did not attach to the surface, (Fig. 1a), and that a non-linear increase in the surface coverage could be observed when increasing the number of porphyrin anchors per origami (Fig. 1b-d).

Thereafter, a ring of $210 \mu \mathrm{m}$ in diameter and $1 \mu \mathrm{m}$ in width was patterned on the plain Teflon AF film using electron beam lithography. The exposed areas feature $20 \%$ reduced hydrophobicity. ${ }^{22}$ The TIRF image in Fig. 1f shows the surface coverage after deposition of fluorophore-modified DNA origamis with 5 porphyrin anchors, which clearly indicates that the e-beam exposed areas of reduced hydrophobicity further promote the adhesion of the origami structures. 

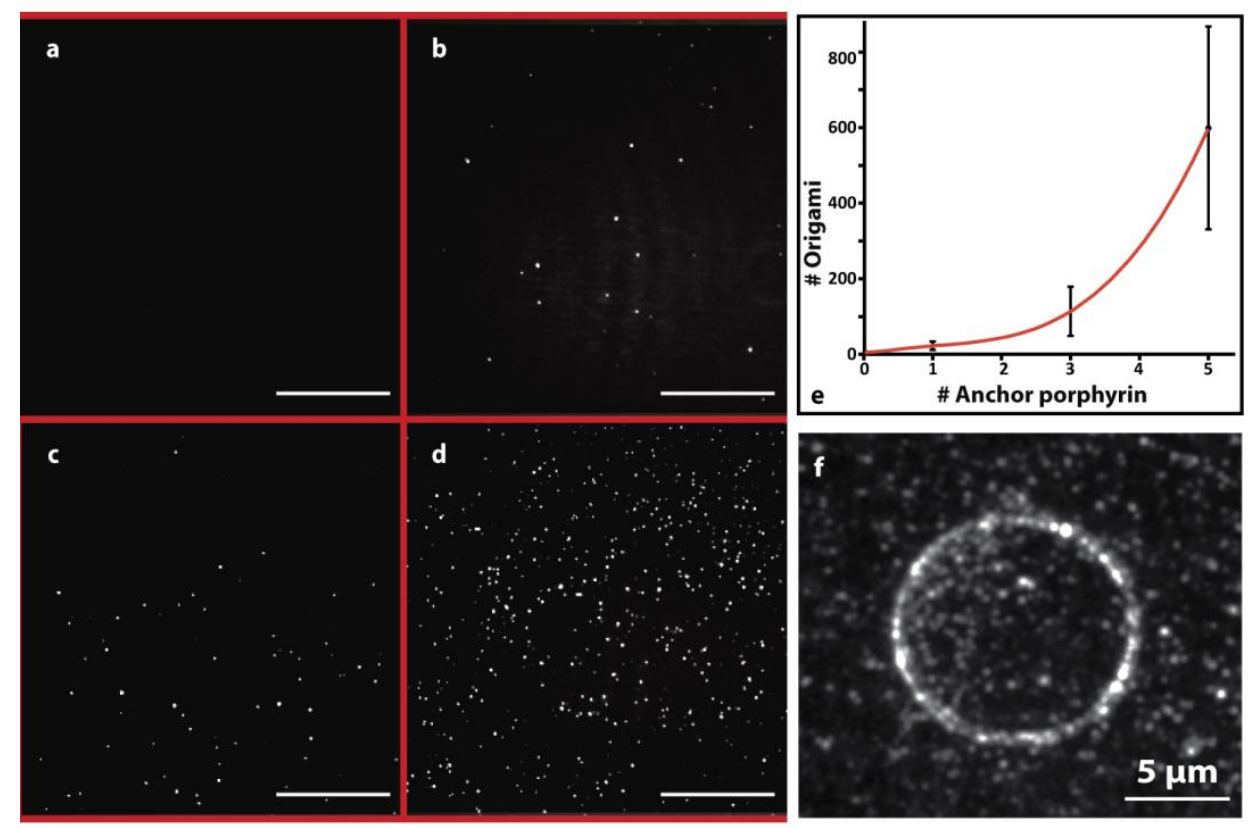

Figure 1 a-d) Total internal reflection fluorescence (TIRF) images of surface coverage for modified DNA origamis with varying number of porphyrin anchors attached to unpatterned Teflon AF. Scale bar: $25 \mu \mathrm{m}$. a) No porphyrin, b) one porphyrin, c) three porphyrin, and d) five porphyrin anchors. e) Number of bound DNA nanostructures per $40 \mu \mathrm{m} \times 40 \mu \mathrm{m}$ area of planar Teflon AF as a function of the number of porphyrins per structure. The red line is a guide for the eye. f) TIRF image of surface coverage of DNA origami modified with 5 porphyrins $(40 \mathrm{pM})$ on an e-beam patterned Teflon AF surface.

Considering that e-beam irradiation causes chemical change at exposed regions, ${ }^{22}$ the improved interaction of the origami with the patterns is attributed to the presence of acid fluoride $(-\mathrm{CF}=\mathrm{O})$ groups that formed upon e-beam exposure. ${ }^{31}$ This is supported by the observation that adhesion of origami improves with increasing exposure dose of the ebeam radiation, which implies that the simultaneous, dose-independent surface potential change, also caused by the e-beam exposure, cannot be the main governing factor in the adhesion process. ${ }^{22}$

\section{Characterization of three individual fluorophore positions in an immobilized DNA}

\section{origami}

The origamis, which were attached to the Teflon surface with 5 porphyrin anchors, were functionalized with three ATTO $647 \mathrm{~N}$ fluorophores in pre-determined positions, assisted by the means of the SARSE (semi-automated RNA sequence editor) program. ${ }^{30}$ A direct 
stochastic optical reconstruction microscopy (dSTORM) image analysis was performed to confirm that the fluorophores are positioned as expected, according to the pre-designed model (see Supplementary information SI2). These fluorophores represent potential functional units that could be attached to defined positions within the DNA origami for desired applications.
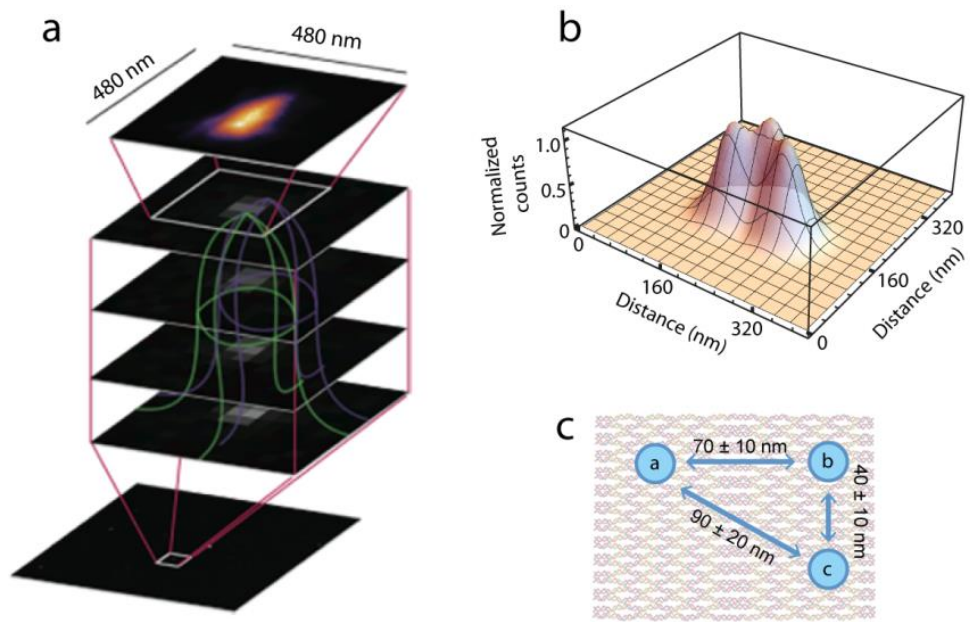

Figure 2 a) Illustration of the direct stochastic optical reconstruction microscopy (dSTORM) analysis principle for individual DNA origami labeled with two fluorophores ATTO $647 \mathrm{~N}$, and anchored to Teflon AF surfaces. b) Gaussian fits for DNA origamis labeled with 3 fluorophores. c) Average distances between 3 fluorophores on a DNA origami anchored to Teflon AF. The uncertainty is calculated based on 20 separate measurements.

Figure $2 \mathrm{c}$ shows a sketch of the rectangular DNA origami with the distances between three ATTO $647 \mathrm{~N}$ dyes positioned in a right-angle triangle. Based on the dSTORM analysis, the distances between the two vertical fluorophores (b-c on Fig. 2c) and two horizontal fluorophores (a-b on Fig. 2c) were $40 \pm 10 \mathrm{~nm}$ and $70 \pm 10 \mathrm{~nm}$, respectively, while the engineered distances were $39 \mathrm{~nm}$ and $73 \mathrm{~nm}$, based on the SARSE program. Moreover, the distance between the two diagonal fluorophores (a-c on Fig.2c) was calculated as $90 \pm 20 \mathrm{~nm}$ from the dSTORM analysis, which is in agreement with $82 \mathrm{~nm}$ based on the SARSE program. These results indicate that the DNA origami structure is 
not significantly altered by the attachment to the hydrophobic support, allowing for the precise positioning of components within the DNA scaffold. We attribute this to the porphyrin linkers, acting both as anchors and as spacers, supporting the origami in retaining its original structure.

\section{Fabrication of nanostructures with Teflon AF as negative e-beam resist}

To achieve individual patterned units and more controlled deposition of origamis on ebeam exposed Teflon, the unexposed polymer was removed from the exposed substrates. For this, FC-75 was used as a developer for the exposed areas, which enables Teflon AF to be used as a negative tone e-beam resist. Figure 3a shows the AFM topography image of silicon substrate directly after e-beam exposure, but before applying FC-75. The image indicates that the Teflon AF films shrink at the exposed areas between 2-7 nm in a dosedependent manner (cf. Fig. 3e, inset) as earlier reported by Shaali et al. ${ }^{22}$ The second set of AFM images on the same substrate, but after development, confirms that the response of the polymer film is that of a negative tone resist (Fig. 3b). 


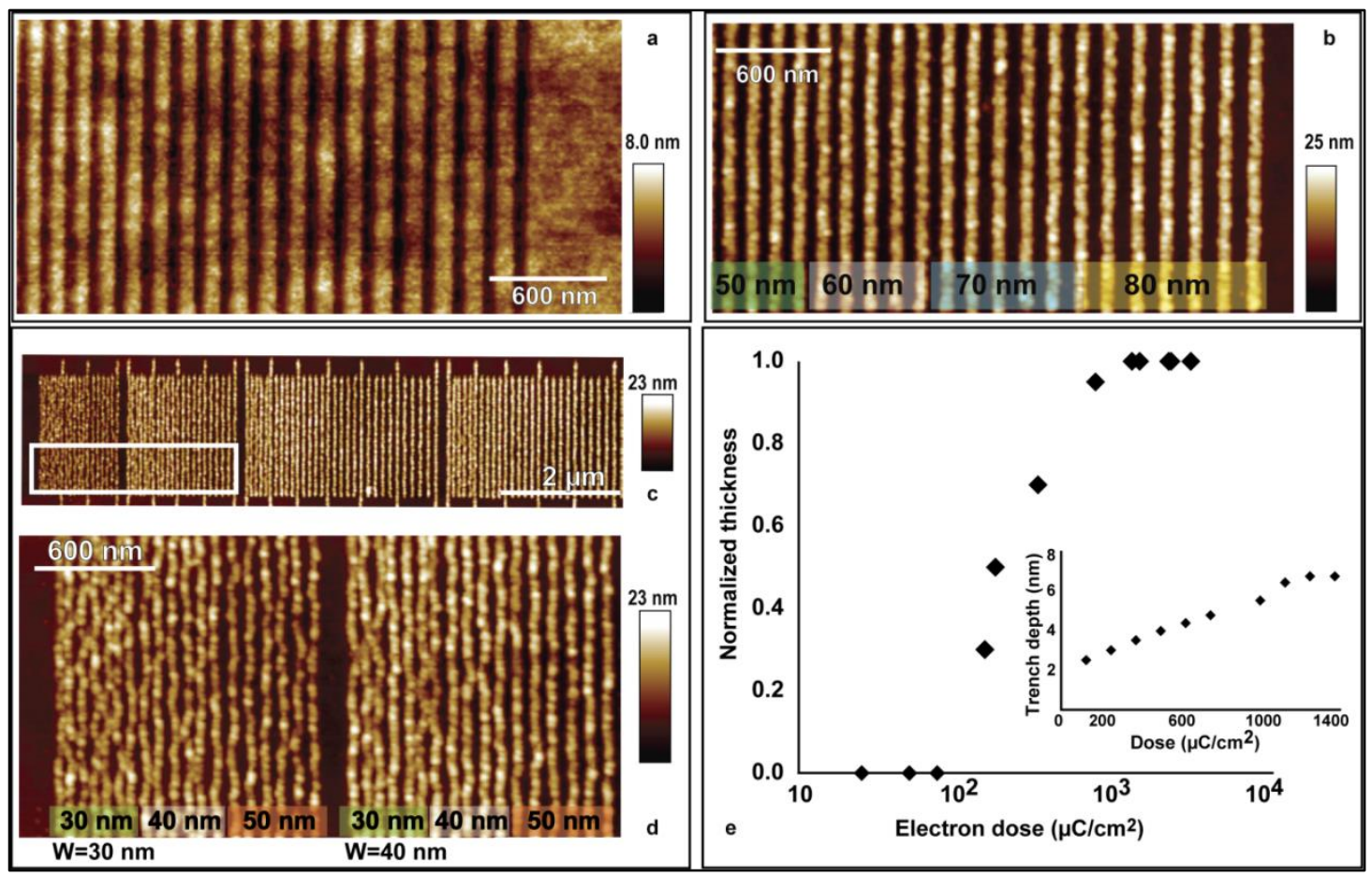

Figure 3 a) Atomic force microscopy image before development showing e-beam exposed line array (line width $70 \mathrm{~nm}$ ), b) Atomic force microscopy image after development on $\mathrm{c}$. The colored fields at the bottom indicate the pitch, c) AFM image of an array of lines exposed to $350 \mu \mathrm{C} / \mathrm{cm}^{2}$ of e-beam radiation, d) an enlarged image showing lines with 30 and $40 \mathrm{~nm}$ in width and different distances, e) The sensitivity curve of Teflon AF, inset) Response of Teflon AF film directly after exposure.

To evaluate the sensitivity of Teflon AF as a resist, an array of dense lines (width of 30 to $70 \mathrm{~nm}$ and pitches of 30-80 nm (Fig. 3c) was exposed using different doses ranging from 25 to $3000 \mu \mathrm{Ccm}^{-2}$. Following the trend of average film thickness after development based on the dose, the contrast of the resist calculated as $\gamma=\left(\log \frac{D_{1}}{D_{0}}\right)^{-1}=1.2$, where $D_{1}$ is the dose at which the resist thickness did not change and $\mathrm{D}_{0}$ is the dose at which the film is removed completely. The sensitivity of $175 \mu \mathrm{Ccm}^{-2}$ was also extracted from the contrast curve (Fig. 3e). By using the optimum dose of electron beam, the smallest feature size was found to be $30 \mathrm{~nm}$ in width and $40 \mathrm{~nm}$ in pitch (Fig. 3d). A minimum time of 2 seconds was required to complete the development step. Beyond that, the development of the features was not time-dependent, therefore no overdevelopment was observed. This is characteristic for most conventional negative resists. 


\section{Positioning of DNA origami on Teflon AF nanopillars}

To localize single DNA origami in predefined surface positions, a square lattice of isolated pillars with the same size as origami and a $1 \mu \mathrm{m}$ pitch was generated on a Teflon AF film (Fig. 4a), using an exposure dose of $\mathrm{D}=1300 \mu \mathrm{C} \mathrm{cm}{ }^{-2}$, followed by development with the perfluorinated solvent. Figure $4 \mathrm{~b}$ shows an AFM image of $10 \times 10 \mu \mathrm{m}$ array of the surface after the development step. The percentage of correctly formed pillars was $>99$ $\%$. Note the displacement of a single pillar structure from its designed position, which is a rare fabrication artifact (yellow circle in Fig. 4b). Binding of the porphyrin-modified, fluorophore-labeled DNA origami to the Teflon AF nanopillars was performed by incubating a DNA origami solution ( $80 \mathrm{pM}$ initial DNA concentration) on the nanopatterned Teflon AF surface, each origami carrying five porphyrin anchors, and in this case for stronger fluorescence signals, five ATTO 647N fluorophores.
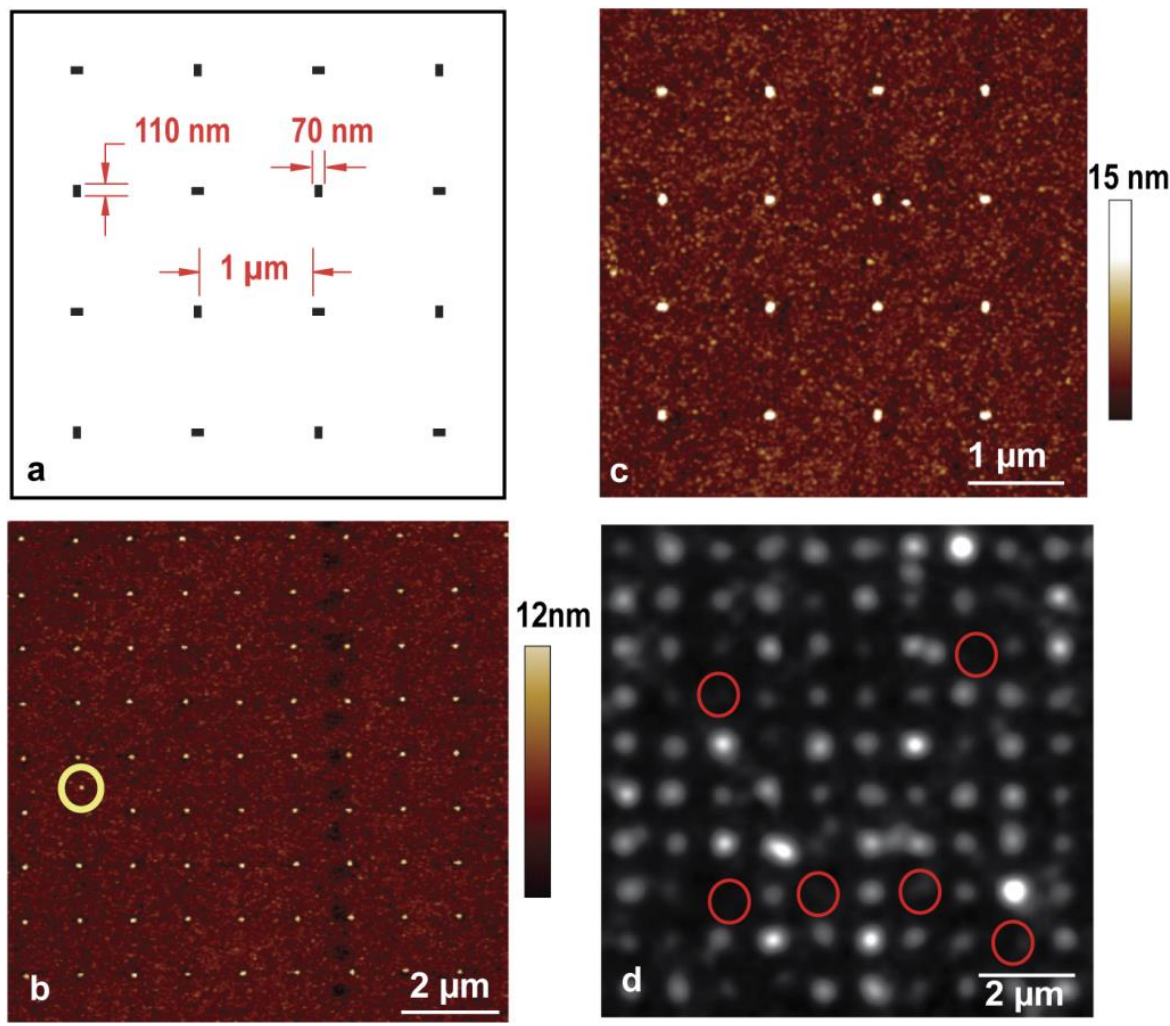

Figure 4 a) Schematic of the nanopattern designed to localize DNA structures. b) AFM micrograph of the surface after e-beam exposure and development of a $35 \mathrm{~nm}$ Teflon AF film. Note the reduced height due to volume shrinking under exposure. c) Enlarged view of (b). d) 
Total internal reflection fluorescence (TIRF) micrograph showing surface coverage after deposition of porphyrin/label-modified DNA assemblies.

The obtained TIRF image (Fig. 4d) shows the preferential binding of the origami to ebeam-treated pillars. A surface coverage of $80 \pm 15 \%$ was achieved as shown Fig. 4d, where the red circles mark sites with missing DNA origami. However, the observed intensity differences (see the intensity histogram in Supplementary Fig. S3) could indicate that there is occasionally more than one origami binding to each pillar. Other reasons for intensity variations could be due to the DNA origamis sitting horizontally on some pillars, and vertically along the sides of some other pillars. Any height difference in fluorophore position can cause different observed intensities due to the evanescent field exponentially decaying with increasing distance from the interface in the TIRF setup. Photochemical bleaching of the fluorophores may also contribute to create the observed intensity differences.

\section{Discussion}

In this study we used DNA origami as a scaffold to accommodate desired molecules, fluorophores in our case, in pre-designed positions within the structure of the DNA strands. We examined the precision of the positioning of the active molecules with direct stochastic optical reconstruction microscopy (dSTORM) image analysis on a Teflon AF substrate. The attachment chemistry of the DNA to the Teflon AF surfaces was provided by porphyrin modified staple strands, which showed irreversible and site-specific binding. The affinity of the DNA to the surface appears to depend on the number of porphyrin anchors present in each origami. Other hydrophobic anchors such as cholesterol might also be suited to immobilize DNA constructs on Teflon AF. ${ }^{32-33}$ Porphyrins and other tetrapyrroles have, however, an additional, distinct application potential for selfassembled light harvesting/energy conversion, ${ }^{34-38}$ and might be capable of enabling the 
surface-based fabrication of biophotonic nanodevices, such as the biomimetic light conversion unit reported earlier. ${ }^{39}$

This study was enabled by Teflon AF as a new negative tone e-beam resist with sensitivity of $175 \mu \mathrm{Ccm}^{-2}$ and resist contrast of 1.2 , capable of providing patterns as small as $30 \mathrm{~nm}$ in width and $40 \mathrm{~nm}$ in pitch. E-beam irradiation of the polymer film causes permanent changes in its chemical structure, leading to a decreased solubility in FC-75 as developer solvent, which is exploited to isolate the written patterns. This resolution is comparable with other common e-beam resists like HSQ (Hydrogen silsesquioxane) at the same acceleration voltage; (20 nm in width and pitches using $7000 \mu \mathrm{Ccm}^{-2}$ on $50 \mathrm{~nm}$ thick films). ${ }^{40}$ The nanostructures obtained by this procedure are highly suitable for microscopy studies. It is noteworthy that the e-beam radiation disintegrates all traces of optically excitable impurities, leading to exceptionally low autofluorescence of the exposed structures.

By combining bottom-up self-assembly and top-down lithography fabrication strategies, we obtained a hierarchically structured system in which we are able to precisely control the positioning of functional units on the molecular level (fluorophores embedded in DNA origamis), and with nanometer precision at predesigned locations on the surface. We believe this new development can become a platform to be used for fabrication of bionanosensors, nanosized light conversion units, or even nano-electronic devices

\section{Methods}

\section{Surface preparation}

Teflon AF is available in two grades, AF1600 and AF2400, which differ by the content of their monomers, and hence in glass transition temperatures of $160{ }^{\circ} \mathrm{C}$, and $240{ }^{\circ} \mathrm{C}$, respectively. They are offered in powder form or as ready-made solutions in a 
commercial perfluorinated solvent such as FC-40, with up to $18 \%$ solid contents. The polymer solutions are typically applied to solid substrates by spin-coating, and then baked above the glass transition temperature to form a homogenous film. Adhesion promoting treatment of the substrate with hexamethyldisiloxane (HMDS) or a perfluorinated organic silane prior to coating is beneficial. ${ }^{29}$ To generate test patterns for characterization, a silicon wafer was rinsed with isopropanol alcohol and MQ water and blow-dried with nitrogen gas. For microscopy purposes ITO-coated microscopy cover glasses can be alternatively used. A thin layer of the adhesion promoter (HMDS, Micro Resist Technology GmbH, Berlin, Germany) was spin coated at $3000 \mathrm{rpm}$ and baked for 5 minutes at $165^{\circ} \mathrm{C}$ on a hotplate. Teflon AF 1600 solution with $6 \%(\mathrm{w} / \mathrm{w})$ solid contents (DuPont Chemicals ,Wilmington, USA) was diluted by FC-40 (Sigma Aldrich, Missouri, USA) to reach $1.2 \%(\mathrm{w} / \mathrm{w})$ in the final solution, and spin coated onto the substrate at $2000 \mathrm{rpm}$. After a baking step at $180{ }^{\circ} \mathrm{C}$ for 5 minutes, the thickness of the Teflon AF film was measured to be $\sim 40 \mathrm{~nm}$ using a surface profiler (Dektak 150 profiler, Veeco instruments, New York, USA). The sample was then patterned by an electron beam lithography system (JEOL JBX-9300FS, JOEL,Tokyo, Japan) at $100 \mathrm{kV}$, using doses in the range of $25-3000 \mu \mathrm{C} / \mathrm{cm}^{2}$. After exposure, the resulting pattern was developed by immersing the substrate in perfluoro-(2-butyl-tetrahydrofuran) (Fluorinert FC-75, TCI, Tokyo, Japan) for 30s under slight agitation. The sample was subsequently blow-dried with nitrogen gas (see Supplementary Fig. S1b). The pattern was characterized using a Dimension ICON scanning probe microscope (Bruker, Masachusettes, USA) in tapping mode, using an NSG15 probe (MicroMasch, South Carolina, USA).

\section{Deposition of DNA nanostructures onto the Teflon AF surface}

For TIRF measurements on plain un-patterned Teflon AF, a deposition concentration of 40 pM DNA origami was used in a buffer consisting of $40 \mathrm{mM}$ Tris-Acetate with 11.5 
$\mathrm{mM}$ magnesium acetate at $\mathrm{pH}$ 8. For TIRF measurements on patterned Teflon AF, a DNA origami solution of $80 \mathrm{pM}$ was used. The deposition protocol consisted of first placing $100 \mu 1$ of DNA solution for 1 minute on the surface, followed by rinsing with fresh buffer for 20 seconds. Finally the sample area was again immersed in buffer, and imaging was performed. The role of the porphyrin anchor in binding to plain un-patterned Teflon AF was studied using origamis with $0-5$ porphyrins and up to 5 ATTO-647N fluorophores. ATTO-647N was placed on the staple strands near the porphyrin modifications pointing in the opposite direction of the origami plane. Accurate placement of ATTO-647N fluorophores was verified using the TIRF microscopy setup and direct stochastic optical reconstruction microscopy (dSTORM) for anchored origamis with 5 porphyrin and 1-3 ATTO-647N fluorophores. The dSTORM analysis was performed following a previously described procedure, ${ }^{41}$ and implemented using in-house made Wolfram Mathematica 8.0 code.

Binding of DNA origami to patterned Teflon AF pillars was investigated using origamis with five porphyrin anchors and five ATTO-647N fluorophores. Excitation was provided by a $641 \mathrm{~nm}$ laser line at $100 \mathrm{~mW}$ (Cube, Coherent, US). Wide-field illumination of the sample was obtained by focusing an expanded beam at the back focal plane of the microscope objective (Leica DM IRB equipped with a 100×, 1.40 NA objective). Emitted light was recorded with an EMCCD camera (DV887, Andor Technology).

\section{dSTORM analysis of DNA origami on Teflon AF}

Under constant illumination, 3000 frames were collected with $100 \mathrm{~ms}$ time averaging, where each pixel corresponded to $160 \mathrm{~nm}$. Frames with total fluorescence intensity from the fluorophores corresponding to a single fluorophore were selected, and the rest were discarded ( 600 frames were left). The program Andor Tracker was used to record all intensity data. Mathematica program was used to fit them in every frame to a $2 \mathrm{D}$ 
Gaussian curve, and to plot the position of the Gaussian maxima in a 2D histogram image. This image was then fitted to the least number of Gaussians possible, which yielded an adequate fit. If this number was below that of the number of fluorophores on the origami, the image was discarded. For three fluorophores the Gaussians were forced to be in a right angle triangle, and the distance between them was fitted.-The stochastic optical reconstruction microscope image (STORM) was fitted using equation 1 and 2.

$$
N_{i j}=\exp \left(-\frac{\left(x-x_{0}\right)^{2}}{2 s^{2}}-\frac{\left(y-y_{0}\right)^{2}}{2 s^{2}}\right) \quad \text { eq. } 1
$$

For a one-dimensional pixel array where $\mathrm{i}$ and $\mathrm{j}$ are the indices of the pixel, $\mathrm{s}$ is the standard deviation of the point spread function, and $\mathrm{N}_{\mathrm{ij}}$ is the expected value from a Gaussian distribution with a width $\mathrm{s}$ and centered at $\mathrm{x}_{0}, \mathrm{y}_{0}$ defined in dimensions of pixels.

$$
\ll \Delta x>^{2}>=\frac{s^{2}+a^{2} / 12}{N}+\frac{4 \sqrt{\pi} s^{3} b^{2}}{a N^{2}} \quad \text { eq. } 2
$$

The resolution was then estimated using equation 2, where $\Delta x$ is the error in localization, $N$ is the number of photons collected (800-1000 in our case), $a$ is the finite size of the pixel, and $b$ is the background noise. Note the importance of collecting multiple photons, $\mathrm{N}$, at each location, as that greatly improves the resolution. Figure $2 \mathrm{~b}$ shows Gaussian fits obtained for one set of data.

Figure $2 \mathrm{a}$ illustrates the principle of the dSTORM analysis for 2 fluorophores on the origami. The bottom micrograph is a raw data frame, with several origamis visible as white dots. Analysis from raw data allows the collection of a set of likely candidates for the accurate position of the ATTO $647 \mathrm{~N}$ dyes on the origamis that are then fitted with a Gaussian spread function model. Four of these candidates are shown above the raw data frame, and the top image shows the summed intensity of all candidate frames. 


\section{References}

1. Rothemund, P.W.K., Folding DNA to create nanoscale shapes and patterns. Nature, 2006. 440(7082): p. 297-302.

2. Ke, Y.G., Douglas, S.M., Liu, M.H., Sharma, J., Cheng, A.C., Leung, A., Liu, Y., Shih, W.M., and Yan, H., Multilayer DNA origami packed on a square lattice. Journal of the American Chemical Society, 2009. 131(43): p. 15903-15908.

3. Dietz, H., Douglas, S.M., and Shih, W.M., Folding DNA into twisted and curved nanoscale shapes. Science, 2009. 325(5941): p. 725-730.

4. Douglas, S.M., Dietz, H., Liedl, T., Hogberg, B., Graf, F., and Shih, W.M., Self-assembly of DNA into nanoscale three-dimensional shapes (vol 459, pg 414, 2009). Nature, 2009. 459(7250): p. 1154-1154.

5. Jungmann, R., Steinhauer, C., Scheible, M., Kuzyk, A., Tinnefeld, P., and Simmel, F.C., Single-molecule kinetics and super-resolution microscopy by fluorescence imaging of transient binding on DNA origami. Nano Letters, 2010. 10(11): p. 4756-4761.

6. Steinhauer, C., Jungmann, R., Sobey, T.L., Simmel, F.C., and Tinnefeld, P., DNA origami as a nanoscopic ruler for super-resolution microscopy. Angewandte Chemie-International Edition, 2009. 48(47): p. 8870-8873.

7. Larsson, A., Carlsson, C., Jonsson, M., and Albinsson, B., Characterization of the binding of the fluorescent dyes yo and yoyo to DNA by polarized-light spectroscopy. Journal of the American Chemical Society, 1994. 116(19): p. 8459-8465.

8. Carlsson, C., Larsson, A., Jonsson, M., Albinsson, B., and Norden, B., Optical and photophysical properties of the oxazole yellow DNA probes yo and yoyo. Journal of Physical Chemistry, 1994. 98(40): p. 10313-10321.

9. Benvin, A.L., Creeger, Y., Fisher, G.W., Ballou, B., Waggoner, A.S., and Armitage, B.A., Fluorescent DNA nanotags: Supramolecular fluorescent labels based on intercalating dye arrays assembled on nanostructured DNA templates. Journal of the American Chemical Society, 2007. 129(7): p. 2025-2034.

10. Ozhalici-Unal, H. and Armitage, B.A., Fluorescent DNA nanotags based on a selfassembled DNA tetrahedron. Acs Nano, 2009. 3(2): p. 425-433.

11. Woller, J.G., Hannestad, J.K., and Albinsson, B., Self-assembled nanoscale DNA-porphyrin complex for artificial light harvesting. Journal of the American Chemical Society, 2013. 135(7): p. 2759-2768.

12. Boeneman, K., Prasuhn, D.E., Blanco-Canosa, J.B., Dawson, P.E., Melinger, J.S., Ancona, M., Stewart, M.H., Susumu, K., Huston, A., and Medintz, I.L., Self-assembled quantum dot-sensitized multivalent DNA photonic wires. Journal of the American Chemical Society, 2010. 132(51): p. 18177-18190.

13. Hannestad, J.K., Gerrard, S.R., Brown, T., and Albinsson, B., Self-assembled DNA-based fluorescence waveguide with selectable output. Small, 2011. 7(22): p. 3178-3185.

14. Kershner, R.J., Bozano, L.D., Micheel, C.M., Hung, A.M., Fornof, A.R., Cha, J.N., Rettner, C.T., Bersani, M., Frommer, J., Rothemund, P.W.K., and Wallraff, G.M., Placement and orientation of individual DNA shapes on lithographically patterned surfaces. Nature Nanotechnology, 2009. 4(9): p. 557-561.

15. Hung, A.M., Micheel, C.M., Bozano, L.D., Osterbur, L.W., Wallraff, G.M., and Cha, J.N., Large-area spatially ordered arrays of gold nanoparticles directed by lithographically confined DNA origami. Nature Nanotechnology, 2010. 5(2): p. 121-126.

16. Penzo, E., Wang, R., Palma, M., and Wind, S.J., Selective placement of DNA origami on substrates patterned by nanoimprint lithography. Journal of Vacuum Science \& Technology B, 2011. 29(6). 
17. Gao, B., Sarveswaran, K., Bernstein, G.H., and Lieberman, M., Guided deposition of individual DNA nanostructures on silicon substrates. Langmuir, 2010. 26(15): p. 1268012683.

18. Resnick, P.R. and Buck, W.H., Teflon ${ }^{\circledR}$ af: A family of amorphous fluoropolymers with extraordinary properties, in Fluoropolymers 2. 2002, Springer. p. 25-33.

19. Resnick, P.R., The preparation and properties of a new family of amorphous fluoropolymers - teflon-af. Advanced electronic packaging materials, ed. A.T. Barfknecht, J.P. Partridge, C.J. Chen, and C.Y. Li. Vol. 167. 1990, Pittsburgh: Materials Research Soc. 105-110.

20. Korinek, P.M., Amorphous fluoropolymers- a new generation of products. Macromolecular Symposia, 1994. 82: p. 61-65.

21. Zhang, H. and Weber, S.G., Teflon af materials, in Fluorous chemistry, I.T. Horvath, Editor. 2012, Springer-Verlag Berlin: Berlin. p. 307-337.

22. Shaali, M., Lara-Avila, S., Dommersnes, P., Ainla, A., Kubatkin, S., and Jesorka, A., Nanopatterning of mobile lipid mono layers on electron-beam-sculpted teflon af surfaces. Acs Nano, 2015. 9(2): p. 1271-1279.

23. Czolkos, I., Hakonen, B., Orwar, O., and Jesorka, A., High-resolution micropatterned teflon af substrates for biocompatible nanofluidic devices. Langmuir, 2012. 28(6): p. 3200-3205.

24. Costela, A., Garciamoreno, I., Florido, F., Figuera, J.M., Sastre, R., Hooker, S.M., Cashmore, J.S., and Webb, C.E., Laser-ablation of polymeric materials at $157 \mathrm{~nm}$. Journal of Applied Physics, 1995. 77(6): p. 2343-2350.

25. Fukutake, N., Miyoshi, N., Takasawa, Y., Urakawa, T., Gowa, T., Okamoto, K., Oshima, A., Tagawa, S., and Washio, M., Micro- and nano-scale fabrication of fluorinated polymers by direct etching using focused ion beam. Japanese Journal of Applied Physics, 2010. 49(6).

26. Katoh, T., Nishi, N., Fukagawa, M., Ueno, H., and Sugiyama, S., Direct writing for threedimensional microfabrication using synchrotron radiation etching. Sensors and Actuators a-Physical, 2001. 89(1-2): p. 10-15.

27. Kobayashi, A., Oshima, A., Okubo, S., Tsubokura, H., Takahashi, T., Oyama, T.G., Tagawa, S., and Washio, M., Thermal and radiation process for nano-/micro-fabrication of crosslinked ptfe. Nuclear Instruments \& Methods in Physics Research Section B-Beam Interactions with Materials and Atoms, 2013. 295: p. 76-80.

28. Miyoshi, N., Oshima, A., Urakawa, T., Fukutake, N., Nagai, H., Gowa, T., Takasawa, Y., Takahashi, T., Numata, Y., Katoh, T., Katoh, E., Tagawa, S., and Washio, M., Nano- and micro-fabrication of perfluorinated polymers using quantum beam technology. Radiation Physics and Chemistry, 2011. 80(2): p. 230-235.

29. Karre, V., Keathley, P.D., Guo, J., and Hastings, J.T., Direct electron-beam patterning of teflon af. leee Transactions on Nanotechnology, 2009. 8(2): p. 139-141.

30. Andersen, E.S., Dong, M.D., Nielsen, M.M., Jahn, K., Lind-Thomsen, A., Mamdouh, W., Gothelf, K.V., Besenbacher, F., and Kjems, J., DNA origami design of dolphin-shaped structures with flexible tails. Acs Nano, 2008. 2(6): p. 1213-1218.

31. Lai, C.Z., Koseoglu, S.S., Lugert, E.C., Boswell, P.G., Rabai, J., Lodge, T.P., and Buhlmann, P., Fluorous polymeric membranes for ionophore-based ion-selective potentiometry: How inert is teflon af? Journal of the American Chemical Society, 2009. 131(4): p. 1598-1606.

32. Erkan, Y., Halma, K., Czolkos, I., Jesorka, A., Dommersnes, P., Kumar, R., Brown, T., and Orwar, O., Controlled release of chol-teg-DNA from nano- and micropatterned su-8 surfaces by a spreading lipid film. Nano Letters, 2008. 8(1): p. 227-231.

33. Borjesson, K., Lundberg, E.P., Woller, J.G., Norden, B., and Albinsson, B., Soft-surface DNA nanotechnology: DNA constructs anchored and aligned to lipid membrane. Angewandte Chemie-International Edition, 2011. 50(36): p. 8312-8315. 
34. Borjesson, K., Woller, J.G., Parsa, E., Martensson, J., and Albinsson, B., A bioinspired self assembled dimeric porphyrin pocket that binds electron accepting ligands. Chemical Communications, 2012. 48(12): p. 1793-1795.

35. Woller, J.G., Borjesson, K., Svedhem, S., and Albinsson, B., Reversible hybridization of DNA anchored to a lipid membrane via porphyrin. Langmuir, 2012. 28(4): p. 1944-1953.

36. Albinsson, B., Hannestad, J.K., and Borjesson, K., Functionalized DNA nanostructures for light harvesting and charge separation. Coordination Chemistry Reviews, 2012. 256(2122): p. 2399-2413.

37. Borjesson, K., Tumpane, J., Ljungdahl, T., Wilhelmsson, L.M., Norden, B., Brown, T., Martensson, J., and Albinsson, B., Membrane-anchored DNA assembly for energy and electron transfer. Journal of the American Chemical Society, 2009. 131(8): p. 2831-2839.

38. Borjesson, K., Wiberg, J., El-Sagheer, A.H., Ljungdahl, T., Martensson, J., Brown, T., Norden, B., and Albinsson, B., Functionalized nanostructures: Redox-active porphyrin anchors for supramolecular DNA assemblies. Acs Nano, 2010. 4(9): p. 5037-5046.

39. Katterle, M., Prokhorenko, V.I., Holzwarth, A.R., and Jesorka, A., An artificial supramolecular photosynthetic unit. Chemical Physics Letters, 2007. 447(4-6): p. 284288.

40. Yang, H.F., Jin, A.Z., Luo, Q., Li, J.J., Gu, C.Z., and Cui, Z., Electron beam lithography of hsq/pmma bilayer resists for negative tone lift-off process. Microelectronic Engineering, 2008. 85(5-6): p. 814-817.

41. Wolter, S., Schuttpelz, M., Tscherepanow, M., Van De Linde, S., Heilemann, M., and Sauer, M., Real-time computation of subdiffraction-resolution fluorescence images. Journal of Microscopy-Oxford, 2010. 237(1): p. 12-22. 